\title{
OUTCOME OF SURGICAL MANAGEMENT OF BIMALLEOLAR FRACTURES IN ADULTS
}

\author{
KRISHNA BHARGAVA VEM ${ }^{1 *}$, ANVESH KUMAR KONDLAPUDI ${ }^{2}$, SAIPAVAN KUMAR MURARI ${ }^{1}$, \\ SRINIVASA MURTHY $\mathbf{D}^{1}$
}

${ }^{1}$ Department of Orthopaedics, Apollo Institute of Medical Sciences and Research, Hyderabad - 500 096, Telangana, India. ${ }^{2}$ Department of Orthopaedics, Sri Devaraj Urs Medical College, Kolar - 563 101, Karnataka, India. Email: krishnabr@live.in

Received: 17 June 2017, Revised and Accepted: 28 July 2017

ABSTRACT

Objectives: Appropriate management of malleolar injuries is crucial as ankle is an important weight-bearing joint, and locomotion is determined by and dependent on joint stability. We studied the functional outcome of surgically managed bimalleolar fractures of the ankle.

Methods: Fractures were classified anatomically and as per Lauge-Hansen, arbeitsgemeinschaft für osteosynthesefragen (AO)/orthopedic trauma association classification. The stability of the structures at the fracture site, the extent of damage, mechanism of injury was assessed. Radiologically, tibiofibular clear space of $>6 \mathrm{~mm}$ and widening of the medial clear space of $>4 \mathrm{~mm}$ were indicators of syndesmotic instability.

All underwent open reduction and internal fixation (ORIF) under spinal and/epidural anesthesia. We used tension band wiring, malleolar screw, Kirschner wires (K-wires), cancellous screws for fixing medial malleolus; one-third tubular plate and K-wires for lateral malleolus. Patients were followed up at 6 weeks for 6 months, evaluated using Biard and Jackson's ankle scoring system.

Results: Of 30 patients, 25 (83.3\%) were men. Right ankle was involved in 56.6\%. Road traffic accident was the cause in $83.3 \% .14$ (46.6\%) had supination external rotation injuries. The AO Type B was the most common (66.6\%). All had a complete union, with a mean time for union 10.6 weeks (8-14 weeks). Functional scores were categorized into excellent (60\%), good (26.6\%), fair and poor ( $\mathrm{n}=02$ each). Superficial and deep infection ( $\mathrm{n}=02$ each), delayed union ( $\mathrm{n}=01$ ) were the complications.

Conclusion: ORIF restores the articular congruity of the ankle joint. Cancellous or malleolar screws are better for fixation of the medial malleolus and lateral plating for fibular fractures.

Keywords: Bimalleolar fracture, Biomechanics, Cancellous screw, Malleolar screws, Open reduction and internal fixation, Plating, Tension band wiring.

(C) 2017 The Authors. Published by Innovare Academic Sciences Pvt Ltd. This is an open access article under the CC BY license (http://creativecommons. org/licenses/by/4. 0/) DOI: http://dx.doi.org/10.22159/ajpcr.2017.v10i11.20808

\section{INTRODUCTION}

Ankle joint is one of the unstable joints in the body due to its anatomic position and is more prone to injury during daily activities. Malleolar fractures are one of the most common fractures in orthopedic traumatology. Treatment of these fractures is complicated and challenging as the outcome influences the locomotion; inadequate and improper treatment may result in long-term orthopedic complications and disability [1,2]. Hence, malleolar fractures require accurate reduction and stable internal fixation similar other intra-articular fractures. Thus, the treatment aims at restoring the joint anatomy and function to the normal or near normal, minimize pain, and other complications.

It is proved that the lateral shift can decrease tibiotalar contact area necessitating proper and perfect anatomic reduction [3]. Operative method restores the anatomy and contact-loading characteristic of the ankle. Surgical intervention can be either closed reduction or open reduction with internal fixation (ORIF). Conservative technique of closed reduction in stable injury yields satisfactory outcome while unstable, displaced, and open fractures need ORIF. The superiority of ORIF over closed treatment has been thoroughly demonstrated in the literature [4]. Additional advantages include easier rehabilitation without a cast, early mobilization and earlier weight bearing [5]. However, the outcome in bimolecular fractures varies. Hence, we assessed the functional outcome and results of surgical treatment of bimalleolar fractures.

\section{METHODS}

This study was conducted by the Department of Orthopedics of a teaching hospital after obtaining clearance from the Institutional Ethics Committee.

Prospective patients were screened after obtaining a written informed consent. Patients aged $>18$ years, diagnosed with a closed bimalleolar fracture of ankle joint, open Gustilo and Anderson fracture Type 1, 2, 3a [6] and signed the informed consent form were included. Those with associated pillion fracture, minimally displaced mono-malleolar fractures, avulsion fractures and stable fractures, and those who were medically unfit for surgery were excluded. Those meeting the selection criteria were included, clinically evaluated by the same orthopedic surgeon.

All patients were assessed clinically, radiologically. The stability of the structures involved at the fracture site, damage to the neighboring structures, mechanism of injury were assessed. Radiologically, tibiofibular clear space of $>6 \mathrm{~mm}$ and widening of the medial clear space of $>4 \mathrm{~mm}$ were considered as indicators of syndesmotic instability.

Fractures of the ankle were evaluated using plain radiographs in anteroposterior, lateral and mortise views. Fractures were classified using the Lauge-Hansen [7], arbeitsgemeinschaft für osteosynthesefragen (AO), ("Association for the Study of Internal Fixation" in English)/orthopedic trauma association system classification systems [8] and anatomical types $[9,10]$. Closed reduction and a below knee posterior plaster of Paris (POP) slab were applied. 
All included patients underwent surgical intervention, i.e., ORIF, and received post-operative care.

Under spinal and/epidural anesthesia, patients were placed in supine position. The ipsilateral buttock was raised on a sandbag for better and clear view of the lateral side. A pneumatic tourniquet was applied in all cases. The procedure was performed in a bloodless field, which facilitated good visibility to describe the fracture pattern and anatomical reduction. Appropriate surgical techniques were applied as per the fracture site. Intraoperatively, the stability was checked by laterally displacing the distal fibula from the tibia and the presence of $>3-4 \mathrm{~mm}$ of lateral shift of talus suggested instability (cotton test) [11]

Parenteral antibiotics were given during the post-operative period for 3-5 days, according to the wound condition. After 10-12 days, sutures were removed, and a below knee cast was applied for 4 weeks. Nonweight bearing gait was started from first or the second post-operative day. Partial weight bearing was started after the removal of the cast (after clinical and radiological signs of union become evident). Active exercises of the ankle were advised. In patients with syndesmotic screw fixation, weight bearing was delayed till screw removal. Followup of cases was done at regular intervals of 6 weeks for a minimum of 6 months and assessed using Biard and Jackson's ankle scoring system [12].

We used tension band wiring (Fig. 1), malleolar screw (Fig. 2), Kirschner wires (K-wires) cancellous screws for fixing medial malleolus. One-third tubular plate (Fig. 3) and K-wires were used to fix lateral malleolus.

Data were captured on the predesigned, pre-approved pro forma and transferred to Microsoft Excel worksheets (2007) and analyzed. Results were expressed as frequency, percentage, range; tables and figures were used to depict the results and explained using descriptive analysis.

\section{RESULTS}

In this 18-month study, we included 30 patients with bimalleolar fracture of the ankle and met the selection criteria. 25 (83.3\%) of our patients were men and only $5(16.6 \%)$ were women. Mean age of the patients was 37.3 years with a range of $19-80$ years. 30 (43.3\%) were in the age group of $19-30$ years, followed by $8(26.6 \%)$ in $41-50$ years age group. Right leg was involved in 17 (56.6\%) and left in 13 (43.3\%) patients. Fractures in $25(83.3 \%)$ cases were due to road traffic accidents, $5(16.6 \%)$ cases were due to fall.

In total, 14 (46.6\%) patients had supination external rotation injuries (SER), and $10(33.3 \%)$ had pronation abduction (PA) injuries (Table 1).

The AO Type B was the most common injury involving 20 (66.6\%) patients, followed by Type C in $8(26.6 \%)$ and was least in Type A $(6.6 \%)$

Patients were operated on days $1-3 ; 14(46.6 \%)$ were operated on day $2,7(23.3 \%)$ on day 3 , and $6(20.0 \%)$ were operated on day 1 . Only 3 patients $(10.0 \%)$ were operated on day 4 or more.

\section{Treatment of individual fractures}

\section{Medial malleolar fracture}

of 30 bimalleolar fractures, those of the medial malleolus was fixed with malleolar screws in $16(53.3 \%)$ patients. $6(20.0 \%)$ were fixed with cancellous screws; $5(16.6 \%)$ with K-wires and $3(10.0 \%)$ were fixed with tension band wiring.

\section{Lateral malleolar fracture}

Of 30 patients, fractures of the lateral malleolar were fixed with onethird tubular plate in $21(70 \%)$ patients. K-wires $(\mathrm{n}=07,23.3 \%)$ and cancellous screws $(n=02,6.6 \%)$ were also used in fixing the fracture.

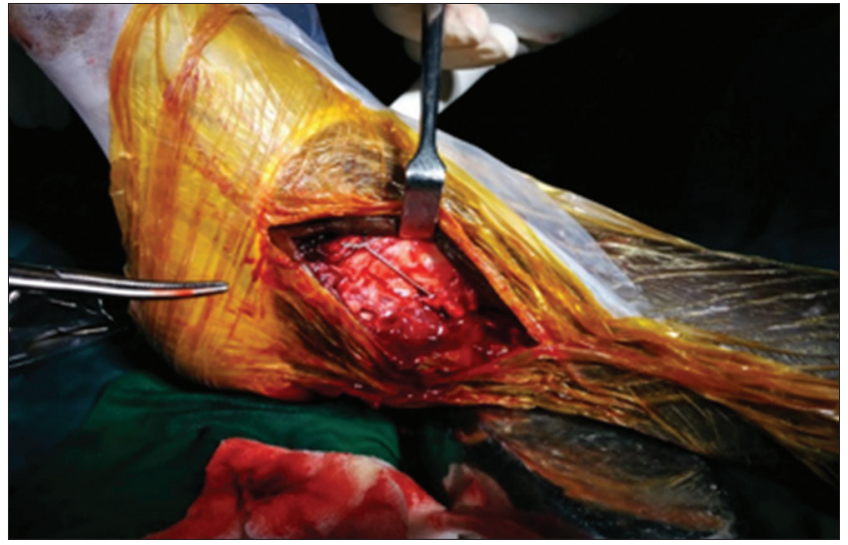

Fig. 1: Tension band wiring for medial malleolus

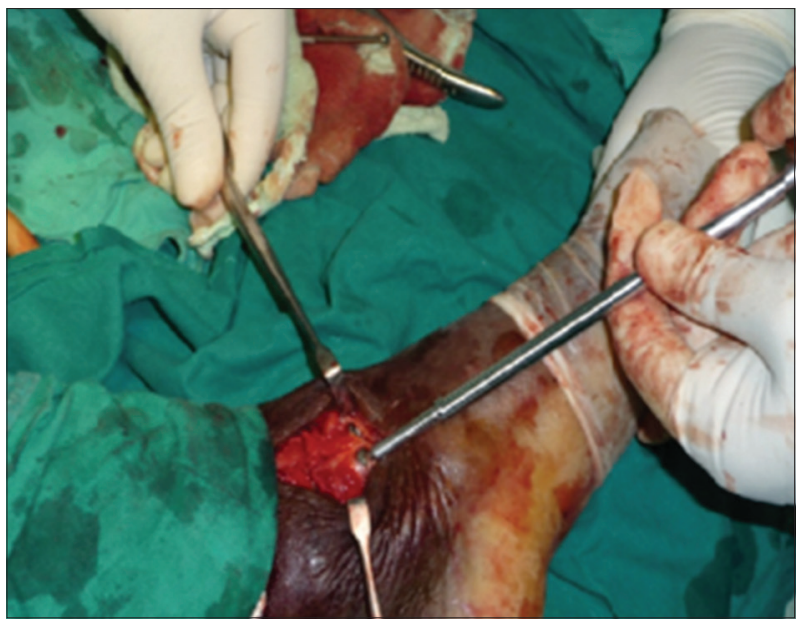

Fig. 2: Malleolar screw for medial malleolus

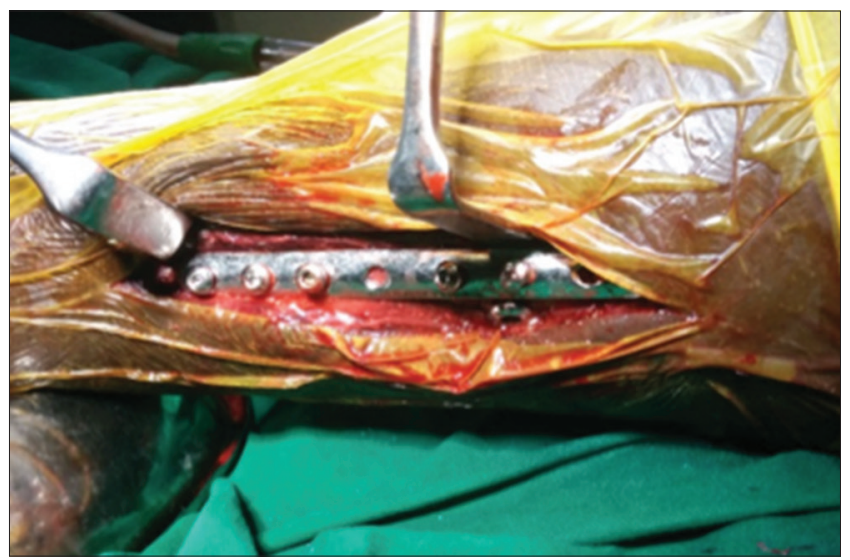

Fig. 3: One third tubular plate for lateral malleolus

Table 1: Classification of injury according to Lauge-Hansen type

\begin{tabular}{ll}
\hline Lauge-Hansen type & n (\%) \\
\hline SER & $14(46.6)$ \\
SA & $3(10.0)$ \\
PA & $10(33.33)$ \\
PER & $3(10.0)$ \\
\hline
\end{tabular}

SER: Supination external rotation, SA: Supination adduction, PA: Pronation adduction, PER: Pronation external rotation 
Syndesmotic fixation

Syndesmotic injury was seen in $3(10 \%)$ requiring fixation with a fully threaded screw. Weight bearing in these cases was delayed till screw removal that was done at 6-8 weeks.

\section{Outcome}

Union

Complete union was seen in all patients, with a mean time taken for the union of 10.6 weeks, and a range of 8-14 weeks (Table 2).

All cases were assessed radiologically before surgery for confirmation, classification and to assess the extent of the injury and postoperatively to assess the outcome, and union (Figs. 4a and b). The union was assessed at regular intervals and during follow-up (Fig. 5).

\section{Functional outcome}

Functional outcome was assessed using Biard and Jackson's ankle scoring system at follow-up. 18 (60.0\%) showed excellent functional score, while $8(26.6 \%)$ had good score. Two patients each showed fair and poor outcome and these patients had an infection. The patients who had poor results had mild pain during their activities of daily living, diminution in their ability to run and do work, reduced motion of ankle, and narrowing of joint space. Figs. 6-8

Table 2: Time taken for complete healing

\begin{tabular}{llllll}
\hline Parameter & \multicolumn{5}{l}{ Time taken for complete union } \\
\cline { 2 - 6 } & $\mathbf{0 8}$ weeks & $\mathbf{1 0}$ weeks & $\mathbf{1 2}$ weeks & $\mathbf{1 4}$ weeks & $\mathbf{1 6}$ weeks \\
\hline Patients & 09 & 10 & 05 & 05 & 01 \\
\hline
\end{tabular}
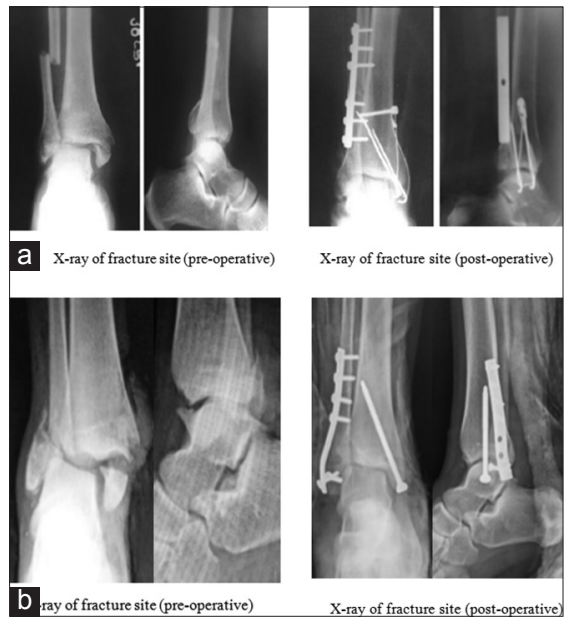

Fig. 4: (a) Patient 1 - X-ray of the fracture site compared pre and post operatively. (b) Patient 2 - X-ray of the fracture site compared pre and post operatively in the same patient

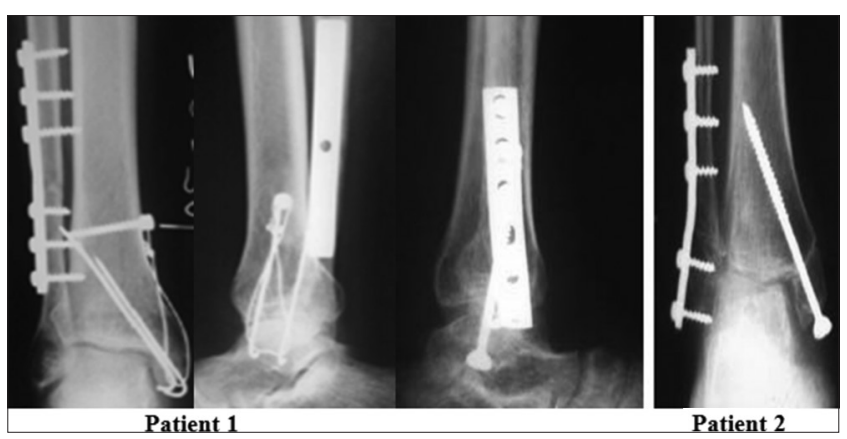

Fig. 5: Complete union at 6 months show the restoration of movement at the ankle joint to the pre-injury level.

\section{Complications}

Complications were seen in $5(16.6 \%)$ patients. Superficial infection and deep infection were seen in two patients each, while one patient had delayed union.

Infections were managed with debridement and administering antibiotics. Delayed union fracture of medial malleolus was treated with continued immobilization, which eventually united without surgical intervention.

Combined functional score was calculated using Biard and Jackson's ankle scoring system and grouped into five categories (A-E). Pain was the common complaint in 17 (56.66\%) patients of whom 15 patients had Grade B, i.e., pain with strenuous activities and only two patients had mild pain with activities of daily living. None of the patients had clinical instability. Following surgery 25 (83.33\%) patients regained the ability to walk desired distance without pain or limp. 17 (56.66\%) patients were able to run desired distances without pain. $12(40.0 \%)$ patients were able to perform their usual occupation without restriction. The range of motion within 10 of the uninjured ankle was seen in $25(83.33 \%)$ patients while $5(16.66 \%)$ had a range within 15 of the uninjured ankle. Anatomical reduction with normal medial clear space and superior joint space was seen in 25 (83.33\%) patients (Table 3 ).
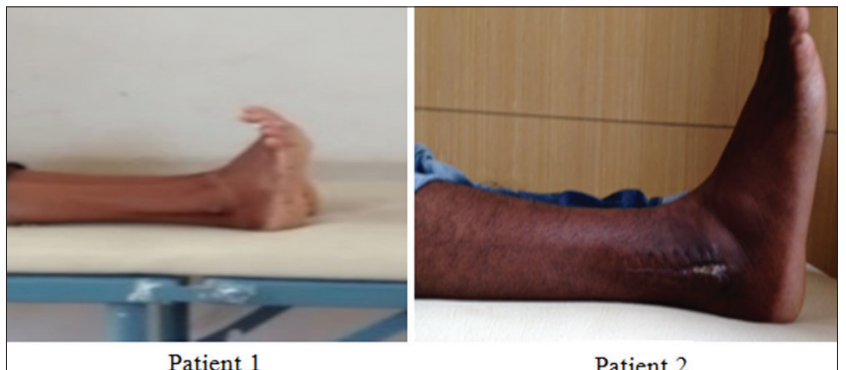

Patient

Patient 2

Fig. 6: Ankle dorsiflexion during follow up

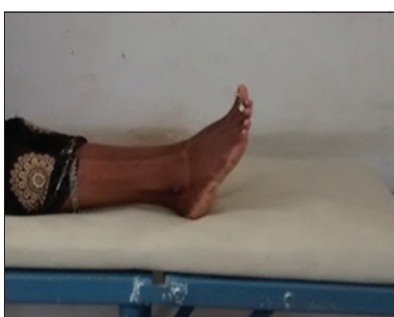

Patient 1

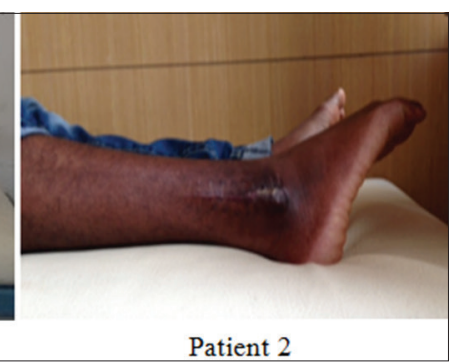

Patient 2
Fig. 7: Ankle plantar flexion during follow up

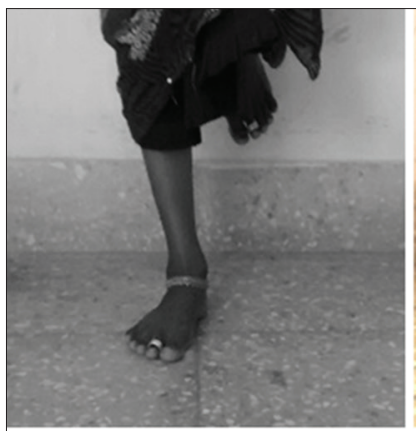

Patient 1

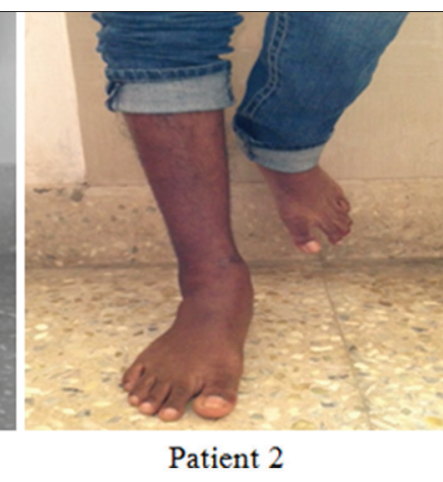

Patient 2
Fig. 8: Restoration of standing on one leg during follow-up 
Table 3: Combined functional scores in different categories (A-E)

\begin{tabular}{llllll}
\hline Category & A & B & C & D & E \\
\hline Pain & 13 & 15 & 02 & 0 & 0 \\
Stability & 30 & 0 & 0 & 0 & 0 \\
Walking & 25 & 03 & 02 & 0 & 0 \\
Running & 17 & 11 & 02 & 0 & 0 \\
Work & 12 & 18 & 0 & 0 & 0 \\
Motion & 25 & 05 & 0 & 0 & 0 \\
Radiographs & 28 & 02 & 0 & 0 & 0 \\
\hline
\end{tabular}

\section{DISCUSSION}

There has been an increase in the prevalence of bimalleolar fractures over the last two decades both in the young, active as well as in elderly patients [3]. Methods adopted to restore the function and prevent arthritis are either closed treatment that includes manipulative reduction and immobilization in the POP cast or ORIF. Burwell and Charnley showed that anatomical reduction and rigid fixation lead to the early return to function [13].

Recent advances have resulted in an evolution in the management strategies of ankle fractures; improved analysis of biomechanics, improvement in fixation techniques and analysis of result of recent studies contributed toward a better surgical outcome. The goal of treatment in these patients is to provide fracture union with a painless full motion of the ankle and with an anatomical restoration of the injured ankle.

Closed method of treatment is often inadequate in restoring the anatomy and biomechanics of ankle in unstable malleolar ankle fractures. Conversely, studies support the ORIF for the restoration of the normal anatomy of the joint provide better results [13-15]

The treatment of malleolar fractures with accurate open reduction and stable internal fixation using AO method and principles has yielded a higher percentage of excellent and good results [2]. We tested this principle on our patients, and our results are in support this principle.

Our patients were younger (mean age of 37.3 years) compared to previous studies (mean age 40-44) [2,12,16,17]. Motwani et al. [18] reported the mean age of their study population as 39.28 years, while Kulloli et al. [19] report a higher mean age of 56.45 years. In contrast, Maruthi et al. report a lower mean age of 28.8 years [20]. Greater proportion (43.3\%) of our study population was in 19-30 years age group. Available reports show male preponderance as in a study by Baird and Jackson (70.0\%) [12] and studies by Indian authors [18-20]; our study population showed male preponderance (83.3\%). However, few studies reported female predominance $[2,16,17]$.

Road traffic injuries are the frequent cause of ankle fracture [17-19], and this is again proven in our study. In contrast, Maruthi et al. [20] report fall as the main cause of fracture, which was the second common cause in our study and in other previous studies [17-19]. Right ankle was the most commonly involved joint in our study similar to the previous reports $[2,16,18,20]$ while; there are reports where in left side was the most commonly involved joint $[12,19]$.

Lauge-Hansen classification system considers the position of the foot and the deforming force that resulted in injury for assessment [21]. We followed this system to classify the ankle trauma. The most common type of injury was SER (46.6\%), followed by pronation-abduction injury $(33.3 \%)$, and our observation is in accordance with previous reports $[12,16,20,22,23]$ Weber classification that considers integrity of syndesmosis, classifies the ankle fracture into three Groups-A, B, C. In our patients, AO Type B was the most common (66.6\%) and least in Type A (6.6\%). Motwani et al. too report a similar observation [18,22,24].
The period between the injury and the surgery is critical and often determines the outcome; surgery after 5 days is associated with delayed bone healing. Delaying surgery often increases the rate of infection. Hence, ideally, these patients should be operated at the earliest [25]. Motwani et al. too operated their patients between days 2 and $5(77.5 \%)$ with a mean time interval of 3.8 days [18]. We operated our patients on day 2 and day $3(70.0 \%)$ while only $10.0 \%$ were operated on day 4 or later. We maintained the time frame well, within the acceptable time period.

All our patients underwent ORIF. We fixed medial malleolar fracture with malleolar screws in $53.3 \%$ patients followed by cancellous screws and K-wires; lateral malleolar fractures were fixed with one third tubular plate in $70 \%$ patients and K-wires in $23.3 \%$. The syndesmotic injury was fixed with a fully threaded screw. These are standard operative techniques followed by orthopedic surgeons worldwide, though preference of the method may vary among the fraternity. We observed better functional outcome in those who underwent stable internal fixation of the medial malleolus by cancellous or malleolar screw, but it was unsatisfactory in those with less rigid fixation using only K-wires. Three of our patients underwent trans-syndesmotic screw fixation. An excellent outcome was seen in one patient, and a good outcome was seen in other two patients.

There is a debate on the effect of fixing of malleolus, on the correction of displacement and stability of talus. Most of them advocate that correction of medial malleolus is sufficient [26] to restore the stability, while others suggest the fixation of the lateral malleolus $[27,28]$. According to Hughes, fixation of the lateral malleolus first, often results in minimal post-operative immobilization and associated with rapid improvement in functions [28].

Time taken for union depends on various factors and has been around 10.4 weeks as reported by Motwani et al. [18] while a longer duration of 13 weeks has been reported by Kulloli et al. [19]. Mean time for the union was 10.6 weeks in our study population with a range of 8-14 weeks, which was similar to the study by Motwani et al. [18].

The success of the surgery is determined by the outcome. Baird and Jackson grading system to describe the outcome is a well-accepted, adopted method and we categorized the outcome in our patients using this grading system. Greater proportion of our patients had good to excellent (86.6\%) outcome compared to reports by Cotton (70.0\%), [11] Beris et al. (74.3\%) [2] and Burwell and Charnley [13], but lesser than that by de Souza et al. (90.0\%) [14] and Maruthi et al. (90.0\%) [20]. Fair outcome varied between 14 and 17\% [2,11,13] which was higher than that reported by de Souza et al. (6.0\%) [14]; we too report a lower rate of fair outcome $(6.6 \%)$. Poor outcome was reported in considerably lesser percentage $(6.6 \%)$ in our patients which was similar to the observations of de Souza et al. (4.0\%) [14], Burwell and Charnley $(6.0 \%) ;[13]$. There are reports of poor response seen in slightly higher proportion of patients varying from $11.1 \%$ to $15 \%[2,11]$; our outcome is similar to those reported by Motwani et al. [18]. Fair to poor results in our patients can be attributed to wound infection and delayed union of the medial malleolus. Restricted activity level and range of movement without radiological evidence of arthritis were noted in four patients.

Infections and delayed healing are the well-documented complications associated with any orthopedic surgeries and surgical maneuver on the ankle joint is no exception to this. Damage to neurovascular structures, superficial and deep infections, scar tenderness, malunion, non-union have been reported as complications of closed reduction [18,19]. We too report infections and delayed union; former were managed with appropriate antibiotic. Complications that we observed are part of any surgical intervention and we managed successfully without much discomfort to the patients.

There is still debate about the duration of immobilisation, but with an inconclusive decision. Few support early mobilization, while 
others argue that immobilization of 4 weeks is required for long-term outcome. We immobilized our patients for 4 weeks, which is a standard protocol. We advised partial weight bearing with an observance of early signs of union radiologically and with complete signs of union, full weight bearing was advised. In those with syndesmotic changes, partial weight bearing was delayed till 6-8 weeks. With our approach of deferring immediate mobilization, our patients did not have instability of the joints (ankle and or subtalar joint) as the soft tissues around the injured joint had sufficient time to heal. Ankle exercises were started later which was also one reason for the good outcome.

Restoring the range of movement is a challenge in orthopedic surgeries, more so in joint. We achieved a good range of movement in $83.33 \%$ patients. Initially, there was a reduced range, but we observed improvement during the follow-up.

The smaller sample size was the major limitation of our study. We did not study the effect of comorbidities on the outcome of the procedure. Comparing with closed reduction would have been described the outcomes better. Effect of the surgery on the subgroups would have been more appropriate.

The result of our study is in accordance with a similar previous study. ORIF has become the mainstay of treatment for most of the unstable bimalleolar fractures, as these operative methods restores the anatomy, biomechanics, and contact loading characteristics of the ankle.

The study concludes that exercising diligence in the management of ankle fractures, adherence to AO method and principles not only improve the accuracy of ORIF but also yield a higher percentage of the excellent and good result.

\section{CONCLUSION}

Mechanism of injury is essential in deciding the method of treatment and for accurate anatomical reduction and fixation. ORIF restore the articular congruity of the ankle joint. Cancellous screws or malleolar screws are better in internal fixation of medial malleolus compared to $\mathrm{K}$-wires fixation, and lateral plating was the best for fibular fractures. Tension band wiring was done for pronation external rotation and pronation adduction injuries showed promising results comparable to that with screw fixation and also gave lesser reports of skin irritation at the wound site. Chances of non-union due to soft tissue interposition can be avoided by surgical treatment.

\section{REFERENCES}

1. Carragce EJ, Csongradi JJ, Bleck EE. Early complications in the operative treatment of ankle fractures. J Bone Joint Surg $\mathrm{Br}$ 1991;73:79-82.

2. Beris AE, Kabbani KT, Xenakis TA, Mitsionis G, Soucacos PK, Soucacos PN. Surgical treatment of malleolar fractures. A review of 144 patients. Clin Orthop Relat Res 1997;341:90-8.

3. Daly PJ, Fitzgerald RH Jr, Melton LJ, Ilstrup DM. Epidemiology of ankle fractures in Rochester, Minnesota. Acta Orthop Scand 1987;58(5):539-44.

4. Weber MJ. Ankle fractures and dislocations. In: Chapman MW, Madison M, editors. Operative Orthopaedics. $2^{\text {nd }}$ ed., Vol. 3. Ch. 50. Philadelphia, PA: JB. Lippincott Company; 1993. p. 731-48.

5. Geissler WB, Tsao AK, Hughes JL. Fractures and injuries of the ankle. In: Rockwood and Green's Fractures in Adults. $4^{\text {th }}$ ed. Philadelphia, PA: Lippincott Raven; 1996. p. 2201-66.

6. Gustilo RB, Anderson JT. Prevention of infection in the treatment of one thousand and twenty-five open fractures of long bones: Retrospective and prospective analyses. J Bone Joint Surg Am 1976;58:453-8.

7. Lauge-Hansen N. Fractures of the ankle: II. Combined experimentalsurgical and experimental-roentgenologic investigations. Arch Surg 1950;60(5):957-85.

8. Muller ME, Nazarian S, Koch P, Schatzker J. Tibia/fibula. In: The Comprehensive Classification of Fractures of Long Bones. Berlin, Germany: Springer-Verlag; 1990. p. 148-91.

9. Leardini A, O'Connor JJ, Catani F, Giannini S. The role of the passive structures in the mobility and stability of the human ankle joint: A literature review. Foot Ankle Int 2000;21(7):602-15.

10. McGlamry ED, Banks AS, Downey MS, Martin DE, Miller SJ, editors. Ankle fractures: Pathomechanics and treatment. McGlamry's Comprehensive Textbook of Foot and Ankle Surgery. $3^{\text {rd }}$ ed. Philadelphia, PA: Lippincott Williams \& Wilkins; 2001.

11. Cotton FJ. Fractures and Joint Dislocations. Philadelphia, PA: WB Saunders; 1910. p. 549.

12. Baird RA, Jackson ST. Fractures of the distal part of the fibula with associated disruption of the deltoid ligament. Treatment without repair of the deltoid ligament. J Bone Joint Surg Am 1987;69(9):1346-52.

13. Burwell HN, Charnley AD. The treatment of displaced fractures at the ankle by rigid internal fixation and early joint movement. J Bone Joint Surg Br 1965;47(4):634-60.

14. de Souza LJ, Gustilo RB, Meyer TJ. Results of operative treatment of displaced external rotation-abduction fractures of ankle. J Bone Joint Surg Am 1985;67(7):1066-74.

15. Cimino W, Ichtertz D, Slabaugh P. Early mobilization of ankle fractures after open reduction and internal fixation. Clin Orthop Relat Res 1991;267:152-6

16. Roberts RS. Surgical treatment of displaced ankle fractures. Clin Orthop Relat Res 1983;172:64-70.

17. Lee YS, Huang CC, Chen CN, Lin CC. Operative treatment of displaced lateral malleolar fractures: the Knowles pin technique. J Orthop Trauma 2005;19(3):192-7

18. Motwani GN, Shah HD, Chavli VH, Daveshwar RN, Parmar H, Suthar PP. Results of open reduction and internal fixation in closed bimalleolar Pott's fracture of ankle in adults. Int J Med Sci Public Health 2015;4(7):893-900.

19. Kulloli SS, Magdum PB, Naik NP. Evaluation of management of malleolar fractures of ankle joint. IOSR J Dent Med Sci 2012;3:27-31.

20. Maruthi CV, Venugopal N, Nanjundappa HC, Siddalingaswamy MK Bimalleolar fracture of ankle joint managed by tension band wiring technique: A prospective study. Sch J Appl Med Sci 2014;2(1D):428-32.

21. Clare MP. A rational approach to ankle fractures. Foot Ankle Clin 2008;13(4):593-610

22. van Laarhoven CJ, Meeuwis JD, van der WerkenC. Postoperative treatment of internally fixed ankle fractures: A prospective randomised study. J Bone Joint Surg Br 1996;78(3):395-9.

23. Egol KA, Dolan R, Koval KJ. Functional outcome of surgery for fractures of the ankle. A prospective, randomised comparison of management in a cast or a functional brace. J Bone Joint Surg Br 2000;82(2):246-9

24. Makwana NK, Bhowal B, Harper WM, Hui AW. Conservative versus operative treatment of displaced ankle fractures in patients over 55 years of age. A prospective, randomized study. J Bone Joint Surg Br 2001;83(4):525-9.

25. Breederveld RS, van Straaten J, Patka P, van Mourik JC. Immediate or delayed operative treatment of fractures of the ankle. Injury 1988;19(6):436-8

26. Svend-Hansen H, Bremerskov V, Baekgaard N. Ankle fractures treated by fixation of the medial malleolus alone. Late results in 29 patients. Acta Orthop Scand 1978;49(2):211-4.

27. Yablon IG, Heller FG, Shouse L. The key role of the lateral malleolus in displaced fractures of the ankle. J Bone Joint Surg Am 1977;59(2):169-73.

28. Hughes J. The medial malleolus in ankle fractures. Orthop Clin North Am 1980;11(3):649-60. 